\title{
良序列 紧 fts
}

C. K. Wang 1973 年在 JMAA 上定义 了序列紧 $\mathrm{fts}$ (每个不分明集序列有收敛的子 序列), 得到每个 $\mathrm{fts}$ 都是序列紧的病态结 果, 且这种定义不是一般拓扑学中序列紧性 的良扩张.

下面给出良序列紧 $\mathrm{fts}$ 的定义, 它以通 常的序列紧空间为特款.

定义 $X$ 为 fts, 对 $\alpha \in(0,1], X$ 中 每个 $\alpha$ 序列都存在子列具有高度为 $\alpha$ 的极限 点,称 $X$ 为良序列紧 $\mathrm{fts}$.

良序列紧性与良紧性有密切的关系, 且 具有许多好的性质.

定理 1 良序列紧性对分明闭子空间 可遗传。

定理 2 良序列紧 $\mathrm{fts}$ 的每个闭子集， 可取到最大值.

定五 3 良序列紧性为 Fuzzy 拓扑不
变性。

定理 4 良序列紧性是可数可积的.

定理 5 良序列紧性是可商的.

定理 $6 X$ 为良序列紧 $\mathrm{fts}, Y$ 是 $Q-C_{\boldsymbol{I}}$ 和 $T_{1 \frac{1}{2}} \mathrm{fts}$.

(1) 若 $f: X \rightarrow Y$ 连续, 则 $f$ 是闭映射;

(2) 若 $f: X \rightarrow Y$ 是连续的双射, 则 $f$ 是 同胚映射。

定理 $7 Q-C_{I}$ 的良紧 $\mathrm{fts}$ 为良序列 紧 fts.

定理 $8(X, \omega(J))$ 为良序列紧 $\mathrm{fts}$ 的充要条件是 $(X, J)$ 为序列紧空间 (其中 $(X, \omega(J))$ 是由通常拓扑空间 $(X, J)$ 诱 导 的 $\mathrm{fts}$ ). 从而良序列紧性是通常序列紧性的 良记张。

宣立新

（南京化工动力专科学校）

\section{变时滞中立型大系统稳定性}

本文考虑中立型大系统:

$$
\begin{array}{r}
\dot{X}_{i}(t)=A_{i i}(t) X_{i}(t)+\sum_{i=1}^{r} B_{i j}(t) X_{j}(t) \\
+\sum_{i=1}^{r} C_{i j}(t) X_{i}\left(t-\tau_{i}(t)\right) \\
+\sum_{j=1}^{r} D_{i j}(t) \dot{X}_{i}\left(t-r_{i}(t)\right)
\end{array}
$$

的解于 $C^{1}$ 空间指数稳定、渐近稳定、稳定的 条件. 其中 $A_{i i}(t) 、 B_{i j}(t) 、 C_{i j}(t) 、 D_{i j}(t)$ 分别 是 $n_{i} \times n_{i}, n_{i} \times n_{i}$ 阶连续矩阵. $X_{i} \in R^{n_{i}}$, $\sum_{i=1}^{r} n_{i}=n, 0 \leqslant \tau_{i}(t), r_{i}(t) \leqslant \tau$.
定义 1 若存在常数 $d_{i}>0, j \in N=$ $\{1,2 \cdots m\}, \delta>0$, 当 $t \geqslant t$ 。使:

$$
\begin{aligned}
& \sum_{\substack{i=1 \\
j \neq i}}^{m} d_{i}\left|a_{i j}(t)\right|+d_{i} a_{i i}(t)<-\delta \quad i \in N \\
& \text { 或 } \\
& \sum_{\substack{i=1 \\
i \neq j}}^{m} d_{i}\left|a_{i j}(t)\right|+d_{i} a_{i j}(t)<-\delta, \quad i \in N .
\end{aligned}
$$

则称矩阵 $A(t)=\left(a_{i j}(t)\right)_{m \times m}$ 是拟负对角占 优.

引理 设 $P_{i}(t) 、 a_{i j}(t) 、 b_{i j}(t) 、 \lambda_{i}(t) \epsilon$ $C\left[t_{0}-\tau,+\infty\right)$ 且 $a_{i j}(t) \geqslant 0(i \neq j), B \geqslant$ $b_{i j}(t) \geqslant 0, B$ 为正常数, $\lambda_{i}(t)>0$,
通报
1988 年 


$$
\sum_{i=1}^{m} \bar{P}_{i}\left(t_{0}\right)=\sum_{i=1}^{m} \sup _{t_{0}-\tau \leqslant \theta<t_{0}} P_{i}(\theta) \geqslant 0,
$$

若满足:（i）存在常数 $k_{i} \geqslant 0$, 使

$$
\begin{gathered}
k_{i} D_{-} P_{i}(t) \leqslant \lambda_{i}(t) \sum_{i=1}^{m}\left[a_{i j}(t) P_{j}(t)\right. \\
\left.+b_{i j}(t) \bar{P}_{i}(t)\right] \quad i \in N,
\end{gathered}
$$

(ii) 矩阵 $M(t)=\left(a_{i j}(t)+b_{i j}(t)\right)_{m \times m}$ 是拟 负对角占优的. (iii) 存在 $0<\lambda(t) \leqslant \min _{1<i<m}$ $\left\{\lambda_{i}(t)\right\}$ 使 $\int_{t-\tau}^{t} \lambda(s) d s$ 有界, 则存在常数阵。

$$
M(t)=\left(\begin{array}{ccc}
-\varepsilon_{1}(t)+m_{1}\left(\left\|B_{11}(t)\right\|+\left\|C_{11}(t)\right\|\right) & \cdots & m_{1}\left(\left\|B_{r 1}(t)\right\|+\left\|C_{1 r}(t)\right\|\right) \\
\vdots & & \vdots \\
m_{r}\left(\left\|B_{r 1}(t)\right\|+\left\|C_{r 1}(t)\right\|\right) & \cdots & -\varepsilon_{r}(t)+m_{r}\left(\left\|B_{r r}(t)\right\|+\left\|C_{r r}(t)\right\|\right) \\
\left\|A_{11}(t)\right\|+\left\|B_{11}(t)\right\|+\left\|C_{11}(t)\right\| & \cdots & \left\|B_{1 r}(t)\right\|+\left\|C_{1 r}(t)\right\| \\
\vdots & & \\
\left\|B_{r 1}(t)\right\|+\left\|C_{r 1}(t)\right\| & \cdots & \left\|A_{r r}(t)\right\|+\left\|B_{r r}(t)\right\|+\left\|C_{r r}(t)\right\| \\
m_{1}\left\|D_{11}(t)\right\| & \cdots & m_{1}\left\|D_{1 r}(t)\right\| \\
\vdots & & \vdots \\
m_{r}\left\|D_{r 1}(t)\right\| & \cdots & m_{r}\left\|D_{r r}(t)\right\| \\
-1+D_{11}(t) \| & \cdots & \left\|D_{1 r}(t)\right\| \\
\vdots & & \vdots \\
\left\|D_{r 1}(t)\right\| & \cdots & -1+\left\|D_{r r}(t)\right\|
\end{array}\right)_{2 r \times 2 r}
$$

则系统 (1) 的平凡解于 $C^{1}$ 上指数稳定.

定王 2 若定理 1 中条件 $1^{\circ}$ 满足, 且 $2^{\circ}$ 存在 $0<\varepsilon(t) \leqslant \min _{1<i<r}\left\{\varepsilon_{i}(t), 1\right\}$

$$
\int_{t-\tau}^{+} \varepsilon(s) d s \text { 有界, } \int_{t_{0}}^{\infty} \varepsilon(t) d t=+\infty .3^{\circ}
$$
$\widetilde{M}(t)$ 是拟负对角占优矩阵

定理 1 若系统 (1) 满足: $1^{\circ}$ 存在连 续函数 $\varepsilon_{i}(t)>0$, 常数 $m_{i}>0$ 使 $\left\|\Phi_{i}(t, s)\right\| \leqslant$ $m_{i} e^{-\int_{s}^{s_{i}} s_{i}\left(t_{1}\right) d t_{1}}>s, \Phi_{i}\left(t, t_{0}\right)$ 是 $\dot{X}_{i}=A_{i i}(t) X_{i}$

$2^{\circ} M(t)$ 是 $2 r \times 2 r$ 拟负对角占优矩

$$
\begin{aligned}
& \tilde{M}(t)=\left(\begin{array}{ccc}
-1+\frac{m_{1}}{\varepsilon_{1}(t)}\left(\left\|B_{11}(t)\right\|+\left\|C_{11}(t)\right\|\right) & \cdots & \frac{m_{1}}{\varepsilon_{1}(t)}\left(\left\|B_{1 r}(t)\right\|+\left\|C_{1 r}(t)\right\|\right) \\
\vdots & & \vdots \\
\frac{m_{r}}{\varepsilon_{r}(t)}\left(\left\|B_{r 1}(t)\right\|+\left\|C_{r 1}(t)\right\|\right) & \cdots & -1+\frac{m_{r}}{\varepsilon_{r}(t)}\left(\left\|B_{r r}(t)\right\|+\left\|C_{r r}(t)\right\|\right), \\
\left\|A_{11}(t)\right\|+\left\|B_{11}(t)\right\|+\left\|C_{11}(t)\right\| & \cdots & \left\|B_{1 r}(t)\right\|+\left\|C_{1 r}(t)\right\| \\
\vdots & & \vdots \\
\left\|B_{r 1}(t)\right\|+\left\|C_{r 1}(t)\right\| & \cdots & \left\|A_{r r}(t)\right\|+\left\|B_{r r}(t)\right\|+\left\|C_{r r}(t)\right\|
\end{array}\right. \\
& \rightarrow \begin{array}{ccc}
\frac{m_{1}}{\varepsilon_{1}(t)}\left\|D_{11}(t)\right\| & \cdots & \frac{m_{1}}{\varepsilon_{1}(t)}\left\|D_{1 r}(t)\right\| \\
\vdots & & \vdots \\
\frac{m_{r}}{\varepsilon_{r}(t)}\left\|D_{r 1}(t)\right\| & \cdots & \frac{m_{r}}{\varepsilon_{r}(t)}\left\|D_{r r}(t)\right\| \\
-1+\left\|D_{12}(t)\right\| \cdots & \left\|D_{1 r}(t)\right\| \\
\vdots & & \vdots \\
\left\|D_{r 1}(t)\right\| & \cdots & -1+\left\|D_{r r}(t)\right\|_{2 r \times 2 m}
\end{array}
\end{aligned}
$$


则系统 (1) 的平凡解于 $C^{1}$ 上渐近稳定.

定理 3 若定理 2 中 $1^{\circ} 、 3^{\circ}$ 满足且存在 $0<\varepsilon(t)<\min _{1<i<r}\left\{\varepsilon_{i}(t), 1\right\}, \int_{t_{0}}^{\infty} \varepsilon(t) d t<+$ $\infty$, 则系统 (1) 的平凡解于 $C^{1}$ 上稳定.

肖冬梅

(华中师范大学数学系,武昌)

\section{伪 脐 曲面的全曲 率}

众所周知, 欧氏空间 $E^{n}$ 中极小曲面的全 面率等于其高斯映射像的体积的 -1 倍. 最 近，陈志华教授分别对球空间 $S^{n}$ 和伪球空间 $H^{n}$ 中极小曲面建立了类似结果 (科学通报, 31(1986)，1:10-13). 设 $M$ 是 $n$ 维黎曼流形 $N^{n}$ 中曲面. 设 $h$ 是 $M$ 的第二基本形式, $H$ 是 平均曲率向量, $\langle$,$\rangle 为 N^{n}$ 的内积. 如果存在 $M$ 上一函数 $\lambda$ 使得

$$
\langle h(X, Y), H\rangle-\lambda\langle X, Y\rangle
$$

对 $M$ 上所有切向量 $X$ 和 $Y$ 成立, 那么 $M$ 叫 $N^{n}$ 中伪脐曲面. 从定义易见，极小曲面是伪脐 曲面。本文我们分别对欧氏空间 $E^{n}$ 、球空间 $S^{n}$ 和伪球空间 $H^{n}$ 中伪脐曲面建立类似结果 (定理 1 、定理 2 和定理 3 )。我们还给出一类 伪脐曲面的例子,即所谓迷向曲面 (定理4).

定理 1 假设 $x: M \rightarrow E^{n}$ 是定向伪脐 曲面, $g: M \rightarrow G r(n, 2)=O(n) / O(2) \times$ $O(n-2)$ 是与 $x$ 相伴的高斯映射. 则有

$M$ 的全曲率: $=\int_{M} K d v=-\operatorname{Vol} \cdot(g(M))$.

$$
+2 \int_{M} H^{2} d v \text {. }
$$

定理 2 假设 $x: M \rightarrow S^{n} \subset E^{n+1}$ 是定
向伪脐曲面, $g: M \rightarrow G r(n+1,2)=O(n+$ 1) $/ O(2) \times O(n-1)$ 是与 $x$ 相伴的广义高 斯映射, 则有

$$
\begin{aligned}
M \text { 的全曲率: }= & \int_{M} K d v \\
= & -\operatorname{Vol} .(g(M))+2 \int_{M} H^{2} d v \\
& +2 \operatorname{Vol} .(M) .
\end{aligned}
$$

定理 3 假设 $M$ 是定向曲面， $x: M \rightarrow$ $H^{n} \subset R_{1}^{n+1}$ 是 $H^{n}$ 的伪脐曲面, $g: M \rightarrow G r^{*}(n+$ $1,2)=L(n+1,1) / O(2) \times L(n-1,1)$ 是与 $x$ 相伴的广义高斯映射, 则有

$$
\begin{aligned}
M \text { 的全曲率: }= & \int_{M} K d v \\
= & -\operatorname{Vol} .(g(M))+2 \int_{M} H^{2} d v \\
& -2 \operatorname{Vol} .(M) .
\end{aligned}
$$

注意: 当伪脐曲面退化为极小曲面时, 定理 1 退化为熟知结果, 定理 2 和定理 3 即 为陈志华教授的结果.

定理 4 如果 $M$ 是 $n$ 维黎曼流形 $N^{n}$ 中迷向曲面, 那么 $M$ 必为伪脐曲面.

李海中

(郑州大学数学系)

\section{某类连续介质力学正则形式场方程及对称守恒性}

文献 [1] 建立了至少能模拟力～热～电 赭合系绕，并具有扩大模拟范围意义的某类 连续介质力学 Lagrange 型场方程

$$
(\partial L / \partial X)^{T}+A_{11}\left[\partial L / \partial\left(A_{11} X\right)\right]^{T}
$$

$$
-(\partial L / \partial \dot{X})^{\cdot T}=0,
$$

其中 $X$ 为场变量矢列, $L\left(X, A_{11} X, \dot{X}, x_{\alpha}\right)$ 为 Lagrange 函数, $A_{11}$ 为关于三维直角坐标系 $x_{i}$ 的矩阵微商算子. 记 $x_{a}=\left(x_{i}, r\right),()_{\alpha}=$ 